Research Article

\title{
The Fusion of Eye Movement and Piezoelectric Sensing Technology Assists Ceramic Art Process Optimization and Mechanical Characterization
}

\author{
Xiaoying Li $\mathbb{D}^{1},{ }^{1}$ Yanglin Zhou $\mathbb{D}^{1},{ }^{1}$ and Yanling $\mathrm{He} \mathbb{D}^{2}$ \\ ${ }^{1}$ School of Industrial Design, Hubei University of Technology, Wuhan 430068, China \\ ${ }^{2}$ College of Design, Guangxi Arts University, Nanning 333403, China
}

Correspondence should be addressed to Yanglin Zhou; 201810962@hbut.edu.cn

Received 13 October 2021; Revised 23 November 2021; Accepted 9 December 2021; Published 30 December 2021

Academic Editor: Guolong Shi

Copyright (c) 2021 Xiaoying Li et al. This is an open access article distributed under the Creative Commons Attribution License, which permits unrestricted use, distribution, and reproduction in any medium, provided the original work is properly cited.

\begin{abstract}
Ceramic product shape evaluation is an important part of product development, an important method to optimize product shape design, and is of great significance to reasonably locate users' consumption psychology and promote the development of ceramic product industry. In this paper, we propose an eye-tracking-based evaluation method for ceramic products from the user's point of view, in view of the fact that there are few studies on ceramic product shape evaluation, and it is mainly led by designers and enterprise leaders subjectively, with low user participation and lack of objective evaluation means and objective data support. In this paper, through the implementation of eye-movement experiments, we obtain and analyze the eye-movement data related to the semantic perception evaluation of product modeling and the overall evaluation of modeling, establish the mapping relationship between user evaluation and eye-movement data, and provide objective data support for modeling evaluation. This paper provides an objective data support for the styling evaluation. This paper provides new ideas for the ceramic product modeling evaluation method, which helps to promote the development of ceramic product industry, improve the brand recognition of enterprises, and help the marketing personnel to make reasonable marketing planning plans. For the semantic perceptual evaluation of ceramic product styling based on eye-tracking, the effectiveness of product styling design concept communication is evaluated. Ceramic products are constantly changing and developing, with new shapes appearing and old shapes being eliminated. Continual innovation and development of ceramics based on inherited traditions can give them a new look and color under the existing modeling style. Compared with other categories, although ceramic modeling has relatively abstract formal characteristics, but it is the extension of the modeling, still has obvious morphological characteristics, and the impact on people's aesthetic mood.
\end{abstract}

\section{Introduction}

Ceramic products have a long history, and people started making ceramic products long ago, which are not only practical but also highly artistic and ornamental [1]. The Chinese ceramic industry has a long and illustrious history, with pottery appearing in China some 5,000 years ago, and early porcelain in the middle of the Shang Dynasty, and in the Ming and Qing Dynasties, ceramic products were exported overseas, spreading and promoting the traditional culture of the Chinese nation. Since China's reform and opening up, the ceramic industry has developed rapidly. In order to fur- ther enhance the industry, China has introduced advanced technology to the ceramic industry and made the products have better market satisfaction by continuously improving firing technology and other ways [2]. In addition, on the original technology, China also innovated the relevant processes and gave a lot of new colors in terms of creativity and design, and now, China produces a large number of ceramic products every year and has become the world's largest ceramic producer. With the development of social and economic development and living conditions, user needs have also increased. According to Maslow's pyramid of needs, people in the case of meeting the needs of the 
bottom gradually expect the satisfaction of the needs of the top [3]. The same applies to the field of products, when the user's bottom demand for products that are functional and easy to use is satisfied, users will seek to meet their needs of high-level products. Therefore, the function of ceramic products, form, structure, and its spiritual and cultural connotations needs to be updated to continue to meet the user's product consumption needs [4]. In recent years, the ceramic industry has shown diversified and individualized market demands. The status of traditional art form as the dominant "overlord" of the typical style of ceramic products began to shake. Traditional ceramic products are facing a serious test and fierce competition [5].

In this environment, in order to meet market demand, ceramic enterprises also gradually from the "design after the function" to "design after the user." Currently mainly by the designer's subjective domination of ceramic product design, product characteristics are vulnerable to certain restrictions and the lack of communication with the user [6]. The shape of ceramic products is an important carrier for spreading spiritual culture and is one of the important concerns in design. Ceramic product shape evaluation is an important part of the preproduct development, an important method to optimize the product shape design, and is of great significance to reasonably position the consumer psychology of users in order to promote the development of the ceramic product industry [7]. The current ceramic modeling evaluation method needs to be improved; ceramic enterprises urgently need to ensure that the ceramic product quality is good under the premise of improving the attractiveness of ceramic product modeling, enhance brand recognition, so that it has a better competitive ability, and then make it occupy the market advantage [8]. With the change of consumer philosophy, the market demand force of Chinese ceramic products will be further expanded. In the study of the relationship between physiological behavior and user evaluation, Tang Gangbei et al. proposed a selection method based on objective eye-movement test and combined with the physiological data of EEG for the disadvantages of the current car scheme selection, using a computer to present four car design drawings for the subjects, collecting the eye-movement and EEG data when the subjects viewed the pictures, combining the subjective evaluation values of the subjects, analyzing the correlation of the three data, and establishing a multidimensional. This method improves the objectivity of car evaluation [9]. In order to objectively evaluate the car styling design, the eye-movement experiment method was used, and the hot spot map generated when the subjects observed the car pictures was used as the data source; using methods such as color feature extraction, the evaluation index system was established by collecting and processing the hot spot map information and quantifying the picture information into data; the evaluation was defined as a dichotomous problem, and the evaluation model was established using Fisher's discriminant method [10]. They used a combination of subjective scoring method and eyemovement experiment method to collect eye-movement data and subjective evaluation data of users comparing and observing multiple pictures of car styling solutions and used partial least squares method to establish a car styling evaluation model and verify the effectiveness of the model [11].

This paper introduces eye-tracking experiments into ceramic product styling evaluation, collects several eyemovement data from subjects, and uses a combination of subjective and objective methods to obtain relevant experimental data from the evaluation activities of target users, so as to quantify the subjective evaluation of users as objective data and provide objective evaluation methods and objective data support for product styling evaluation. Improve the traditional evaluation method mainly led by designers, provide new ideas for ceramic product shape evaluation, and help promote the development of ceramic product industry. For the characteristics of user, scoring value is 0-10 integer as discrete variables; support vector machine classification method is used to establish the mapping model between eye-tracking experimental data and user scoring value, which can effectively reduce the error and improve the reliability of evaluation results. The eye-tracking technology is used to conduct the semantic perception test of ceramic product modeling, to measure the objective perception of the target users on the product design concept, to provide objective guidance for the expression of modeling design concept and product marketing promotion, to help the enterprise marketers to reasonably optimize the product promotion and marketing plan, to effectively convey the design concept, and to improve the brand recognition of the enterprise.

\section{Related Work}

Ceramic products have a long history in China. In the early days, they were mainly daily necessities, so more attention was paid to their use function [12]. In recent years, as users' emotional needs for products have increased, the shape of ceramic products has gradually received more attention. How to judge whether users can be attracted by the shape of the product and thus influence the purchase decision requires an effective evaluation of the shape of ceramic products from the user's perspective [13]. They established a structural model of ceramic teaware user needs through hierarchical analysis in three directions, appearance, function, and value, used subjective questionnaires to determine the evaluation index weights, and conducted consistency tests on the obtained results, which showed that the tested user group paid more attention to the appearance of ceramic teaware; further used the fuzzy evaluation method to establish a user fuzzy comprehensive evaluation matrix, determine the evaluation criteria, and after calculating Establish a secondary comprehensive evaluation matrix to build a ceramic tea set evaluation system that quantifies the evaluation results [14].

The researchers collected and identified the perceptual vocabulary and the sample map of experimental ceramic products, created a five-order semantic scale for perceptual imagery, obtained the subjective evaluation data of the test subjects, processed the data using factor analysis to obtain the characteristic imagery factor; used the quantitative I class method, created a product morphological element coding 
table, quantified the morphological elements, and used multiple linear regression analysis to quantify the imagery factor and morphological element quantified values [15]. The analysis was performed by using multiple linear regression analysis to obtain the morphological factor category score and analyze the products according to this score. They summarized the advantages of the designed ceramic products from the designer's point of view in four aspects: function, shape, aesthetics, and economy; analyzed the rationality of the product shape structure to verify its usability; analyzed the product contour lines, indicating that the lines have a sense of rhythm and undulation, and considered that the products have clear characteristics and good decorative effects [16]. However, this evaluation method is only from the designer's point of view and lacks real user feedback evaluation. At present, there are fewer studies on the evaluation of ceramic product shape, mostly based on subjective evaluation, lacking objective evaluation methods, and objective data support and mostly from the designer's perspective lack of real user participation in the evaluation.

The concept of semantic perception comes from product semantics and cognitive psychology. Product semantics is the expression of the product's own spiritual connotation and concept conveyed, and thus, product semantic perception is the way users perceive and understand the product's spiritual connotation and design concept [17]. They studied and analyzed automobiles to construct a mapping relationship between users' attention to different areas of the sample and user information, which was used as a basis for optimizing the questionnaire and thus making the analysis of the semantics of styling imagery more scientific and reasonable. The researchers analyzed the source culture, used the semantic difference method to obtain the cultural semantic words, and used the shape grammar to extract the implicit cultural elements of NRMs based on the product DNA theory to build a topologically expandable semantic model together with the explicit elements; they used the graphical thinking to convert the cultural features into graphical semantics to obtain the core NRM cultural semantic set and build a library of elements to help design activities; they used the electric vehicle charging service facility as a practical case to verify the feasibility of the method [18].

The feasibility of the method is verified. The researchers obtained user semantic-aware vocabulary from online reviews, used WordNet to filter high-frequency words, analyzed the association rules of words, used Apriori algorithm to filter words again, and built the seed feature word set from the obtained words; used neural network algorithm to train word vectors, then expanded the seed feature word set by calculating the similarity between vectors, and finally used manual annotation method to classify the words and obtain the product semantics. Finally, the lexicon is classified by manual annotation to obtain a product semantic feature lexicon. Chen et al. used the importance and similarity principles to establish a hierarchical model of porcelain vase modeling, extracted the typical features of each modeling element, and established a database of porcelain vase modeling; used cluster analysis and word frequency statistics to obtain the semantic words for porcelain vase description and then built a mapping inference library of semantic words and porcelain vase modeling; and established a user customization module system, and the system can automatically retrieve the porcelain vase modeling and generate a $3 \mathrm{D}$ model after the user selects the semantic words. The system can automatically retrieve the vase shape and generate the 3D model after the user selects the semantic words [19].

Early applications of eye-tracking technology were mainly used in the field of psychology, but now, it has been extended to a variety of fields including advertising, interface usability, product design, and other related fields and is becoming popular. Researchers have investigated a method to quantify the aesthetic quality of product design by applying eye-tracking technology to analyze subjects' eye movements to predict users' aesthetic preferences and to develop an aesthetic measurement system [20]. By using a wearable eye-tracking instrument, the researchers studied the changes of subjects' eye gaze characteristics when observing different pictures; the experiment used two types of emotional pictures to stimulate the subjects, extracted the subjects' gaze characteristics under each type of stimulation, and analyzed and compared the experimental results; it showed that the gaze characteristics are correlated with individual emotions, and the gaze characteristics can objectively reflect individuals' emotions within a certain range [21]. The researchers studied the eye-movement characteristics of young women when observing advertisements and investigated whether there were differences in eye-movement behaviors among individuals; seven advertisement images were presented to 80 participants using a monitor, and the gaze information was recorded using an eye-tracking device.

\section{Optimization of Ceramic Processes Combining Eye Movement and Piezoelectric Sensing}

3.1. Ceramic Product Process and Mechanical Characteristics. As a cultural form, ceramic art has certain peculiarities, with unique artistic charm and formal characteristics. Ceramic modeling has a distinctive cultural style and is self-contained, containing a deep artistic connotation. Ceramic modeling elements include form, texture, and color. It is not only a reflection of the basic needs of human beings in the development of civilization but also expresses the spirituality of a nation to a large extent. Human beings began to develop ceramic products from very early on; these products were at first to meet the needs of rituals or to meet the needs of people's lives; then, people also began to pour into their own emotions in their works, according to the law of development of things let ceramics and material, spiritual, technical, and other integration, which is the wealth of human beings, but also the embodiment of human wisdom. For the overall evaluation of ceramic product styling based on eye-tracking, eye-tracking experiments are conducted with target users, and users are asked to score the satisfaction of the tested product, multiple eye-tracking data and scoring scale data of all users are collected and collated, a mapping relationship model between eye-tracking data and user 


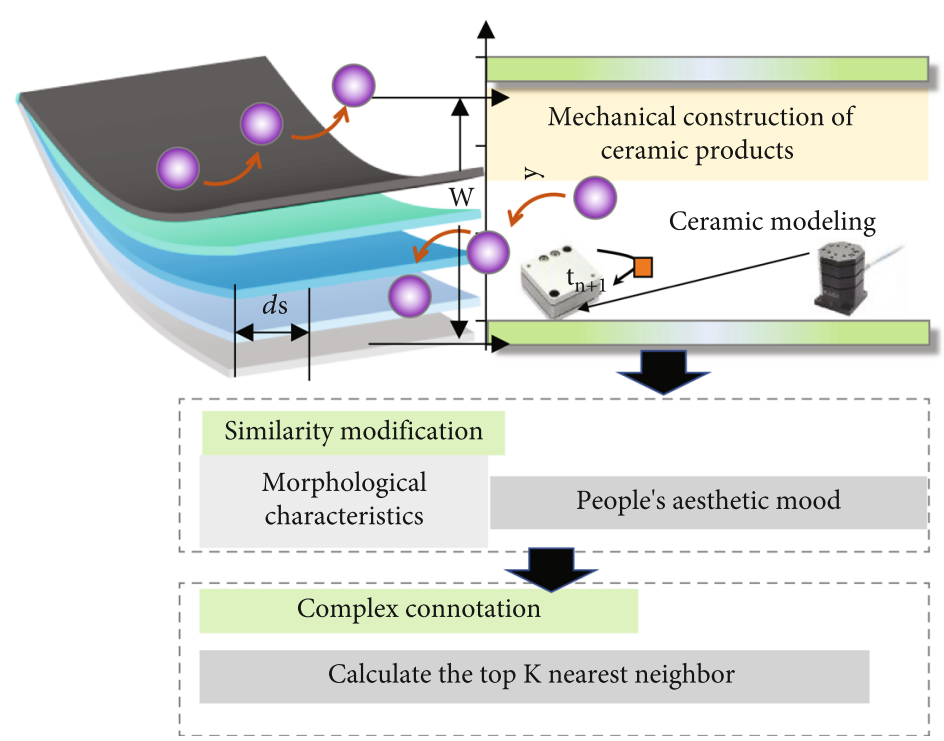

FIgURE 1: Mechanical construction of ceramic products: for static shapes, appreciating the cohesive and expansive sense of form and giving shape changes can make them show a different kind of life in space and volume.

scoring values is established using support vector machine, and finally, the validity of the evaluation method is verified using test set data. Based on the existence of intentions and concepts, the original material is processed to make it into a style and form with certain purposes, and to meet the different needs of culture and art is the ceramic design. The language of ceramics is relatively unique, with its own expression and modeling methods, and unlike other art categories, the language of ceramics is intuitive and does not need to be interpreted as deeply as painting and other art. For ceramic products, by embellishing them and other ways, you can create more layers of connotation from the visual aspect; this perception is not completely unconscious and not completely conscious. For static shapes, appreciating the cohesive and expansive sense of form and giving shape changes can make them show a different kind of life in space and volume, as shown in Figure 1. Ceramic products are constantly changing and developing, with new shapes appearing and old shapes being eliminated. Continual innovation and development of ceramics based on inherited traditions can give them a new look and color under the existing modeling style. In this regard, foreign scholars have pointed out that ceramics is a complex art, which has a simple form, but also needs to have a complex connotation.

3.2. Eye-Tracking-Based Evaluation of Ceramic Products. The subjects are the subjects whose eye movements are tracked by the eye tracking device in the eye tracking experiment, and their eye-movement behaviors can be recorded by the eye tracking device and analyzed and interpreted by the professional eye-movement data collection and analysis software to understand the subjects' psychological cognition and other situations. Depending on the content and method of the eye-tracking experiment, different conditions of subjects are required. Subjects can be classified according to characteristics such as age, gender, profession, and income. According to the requirements of the eye-tracking experi- ment to be implemented, a reasonable selection of subjects to make sure that the subjects meet the requirements of the eye-tracking experiment research is one of the important conditions to ensure the validity of the eye-tracking experiment data. The eye-tracking experiment conducted in this paper is a ceramic product modeling evaluation experiment, and this experiment needs to focus on the user's perception. Therefore, when looking for subjects, it is necessary to first analyze the characteristics of the subjects, classify their characteristics into categories, and then screen them, and select users according to the research objectives, so that they can become subjects of eye-tracking experiments, which is the basic condition for eye-tracking experiments to be meaningful. In order to get the real target users, a detailed analysis of the product is needed to determine the characteristics of the real target users. Usually, the target users of the product are determined at the beginning of the product design, which can be obtained through research and used as the selection criteria for choosing subjects. According to the specific characteristics of the identified target users, screening is conducted according to the age, gender, motivation, and acceptable price level of the users, and interviews are conducted with the users who come to take the test as the basis for screening. In general, real target users who have plans to buy the product will actively seek and think about the product and its related other products of the same type and have some research understanding of them. Therefore, through the interview method, some simple inquiries should be made to the test person, such as the shape, structure, price, and other characteristics of the product and its similar products, to clarify whether he or she has some understanding, to verify whether the test person is the target user, and then to select him or her.

When conducting eye-movement experiments on actual scenes such as display or operation, users usually experience the actual scenes in a fixed environment, so the evaluation they give is also limited to this environment, for example, 

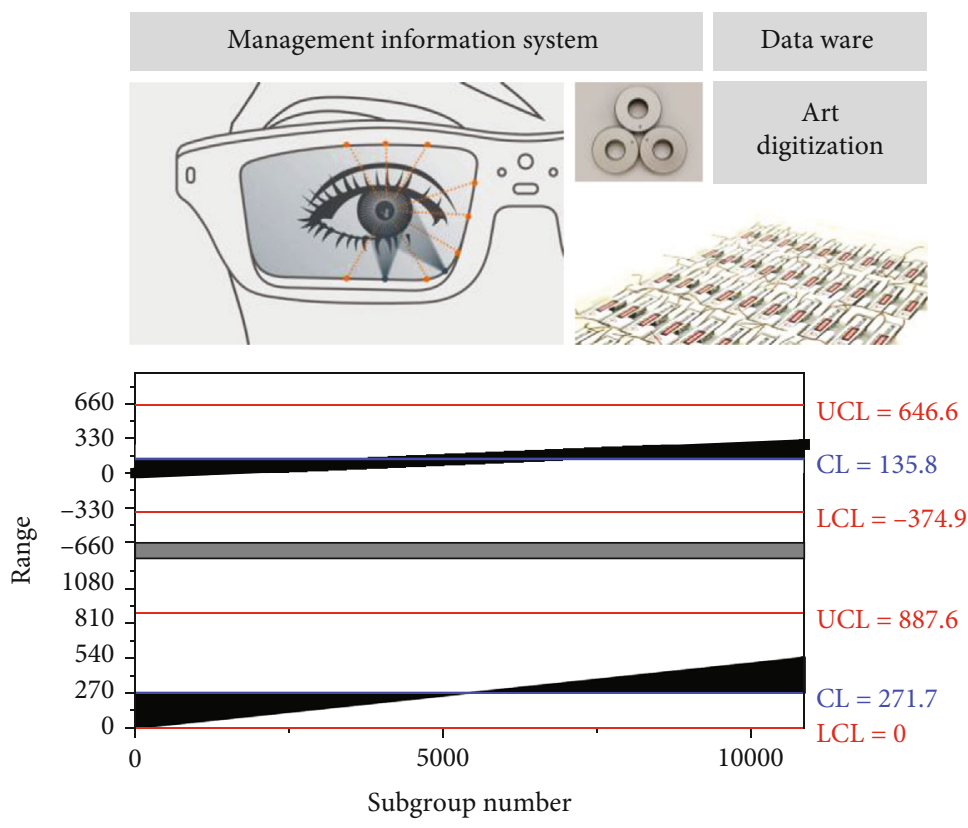

FIGURE 2: Eye-movement experiment.

eye-movement experiments on car driving experience, as shown in Figure 2. In order to make this kind of experiment more effective, if various conditions can be realized, such as test site, test equipment, and product display method, the eye-movement test scenario can be built according to the actual situation. For example, to test whether the product display method can facilitate users to find products, users need to observe the actual product shelves in the field. By wearing a head-mounted eye-tracking device, the subject can get the real experience of looking for the product with as little interference as possible. It should be noted that if detailed and accurate eye-movement data are needed, there are many interfering factors in the actual scenario, such as the movement of eyes relative to the experimental material, light changes caused by position changes during the activity, and subjects being attracted to the sight by nonexperimental factors, which make the experiment generate interfering data and cause experimental errors, and it is difficult to distinguish and reduce such errors in the current study.

If the conditions required for the experiment do not allow, including test equipment, test conditions, and product display methods, that is, the experiment can not be conducted by building the actual experience scene, then the experiment can be conducted by building a virtual experience scene, when the need to use the relevant computer technology and a variety of external sensory equipment to present the combination of experimental materials to build an eye-tracking test scene, as shown in Figure 3. For example, when evaluating multiple product shapes, the subject needs to see all the tested products with the same field of view and adjust the display order of the tested products within a short period of time, which is difficult to achieve in real scenarios. This method is mostly used in current research to implement eye-movement experiments, which

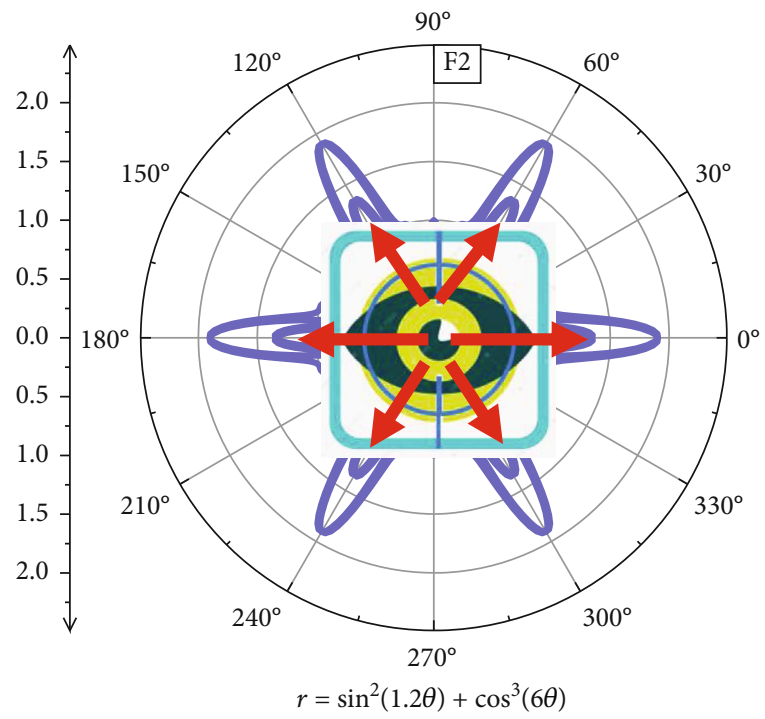

FIgURE 3: Eye-movement frequency tracking.

is widely used and easy to control a variety of variables, more effective, and reliable. In this study, this method was used.

3.3. Eye Tracking Combined with Piezoelectric Sensing Technology. Before building the model, we make the following assumptions: first, it is assumed that there is no debonding and slippage between any interface including the substrate and the skin; second, since the thickness of the piezoelectric layer PZT is only $1.5 \mathrm{gm}$, while the thickness of the substrate is more than $30 \mathrm{~nm}$, a difference of about twenty times. Therefore, the bending deformation energy of the PZT is much smaller than that of the substrate (the bending modulus is proportional to the third power of the thickness). Moreover, the twelve PZT units are concentrated in the center of the arch, and the actual area is much smaller than the 


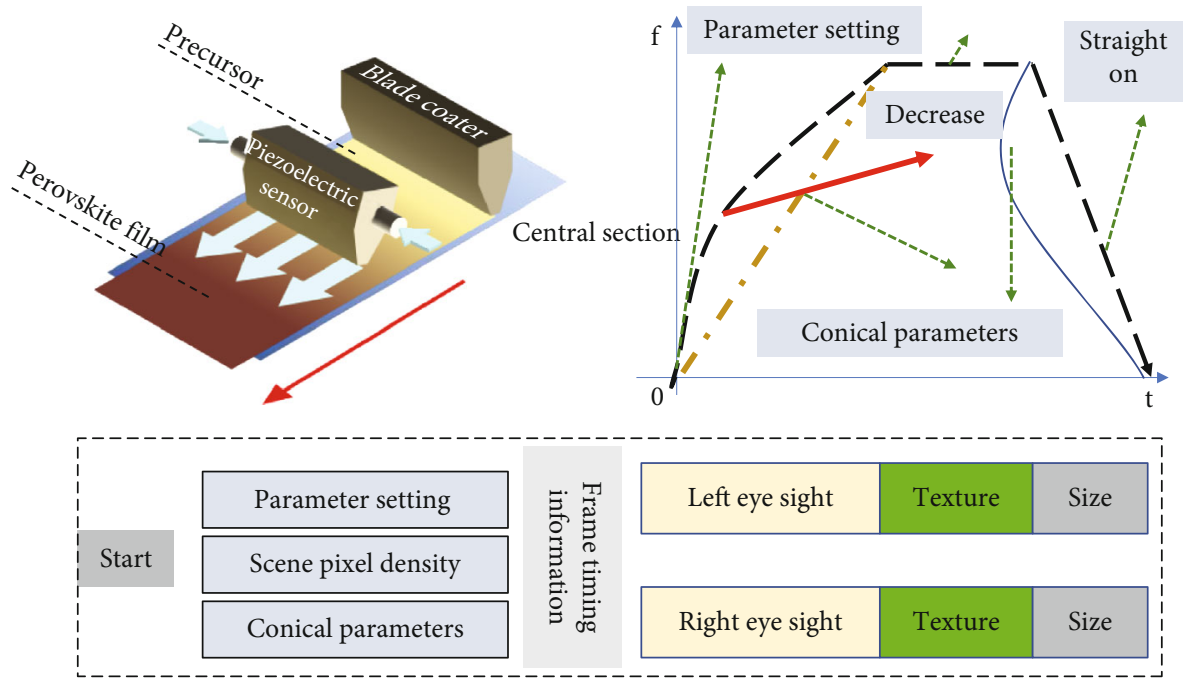

FIgURE 4: Geometric model of the central section of the piezoelectric sensor.

area of the substrate, so the deformation energy of the PZT units themselves and the influence on the substrate are ignored in the calculation.

As in Figure 4, the horizontal direction is the $x$-axis, and the vertical direction is the $z$-axis; The original length of the unfolding level of the device is known, and the displacement occurs after the both ends of the end part are flexed, and the flexural amplitude of the subbase neutral axis at the height of the $x$-coordinate position can be obtained.

After the morphological observation, we assume that the deflection equation of the arch part can be expressed by the cosine function as follows:

$$
w=\frac{q}{l}\left\{x^{2}+\cos 2 \tau\right\}
$$

where $A$ and $L 0$ represent the amplitude of buckling and the original length of the device, respectively. Here, we neglect the bending deformation energy, membrane strain energy, and electric field energy of the PZT itself after which the total energy of the system can be expressed as:

$$
\rho 3-\rho 6 \leq(\operatorname{criT} 1-x) \leq \rho 3+\rho 6,
$$

where $E$ and $h$ are the equivalent Young's modulus Dong and thickness weevil of the combined skin substrate, respectively, and are the relative displacements at the two ends of the device. By taking the derivative of the total energy with respect to the amplitude to zero, the

$$
T_{f}=\frac{n_{i, j}}{\sum_{i=1}^{n} w_{i}} .
$$

The amplitude of flexion can be derived as

$$
Q \sqrt{\left[t^{2}-r\right]}=Q \sqrt{t}+0.1 t^{2}
$$

The strain at the neutral axis of the PZT strip can be derived from the equilibrium equation.

$$
\text { MathExp }=-k \log _{2}\left(\frac{A}{B}\right)-k^{\prime} \log _{2}\left(\frac{A^{\prime}}{B^{\prime}}\right)
$$

Among them.

$$
n=\frac{\left(\rho^{3}-\rho 6\right)}{\left(\rho^{3}+\rho 6\right)},
$$

is the ratio between the bending modulus of the bilayer substrate and the bending modulus of the bilayer substrate plus the PZT functional layer composite structure, while $z$ is the distance between the PZT neutral layer and the neutral layer of the composite structure. Since the length of the PZT strip is much smaller than the length of the entire device and is concentrated in the center of the device, we use an approximation in the derivation of the equation.

$$
0 \leq n \leq 1
$$

\section{Experiments and Result Analysis}

4.1. Sensor Monitoring Results. Figure 5 shows the output voltage of the subject's wedge for different fatigue states acquired by the oscilloscope. Since the oscilloscope was connected to an external power supply, the output signal was interfered with spurious waves, and the above results are the result of filtering the signal above $15 \mathrm{~Hz}$ by using the fast Fourier transform. The blink curves for the first three blinks are similar to the results above, with waveforms in the positive yield range. From the fourth and fifth blinks, it can be seen that the time period after the voltage rises to the peak until the voltage starts to increase in the opposite direction is significantly too long, reaching about $0.5 \mathrm{~s}$. This means 


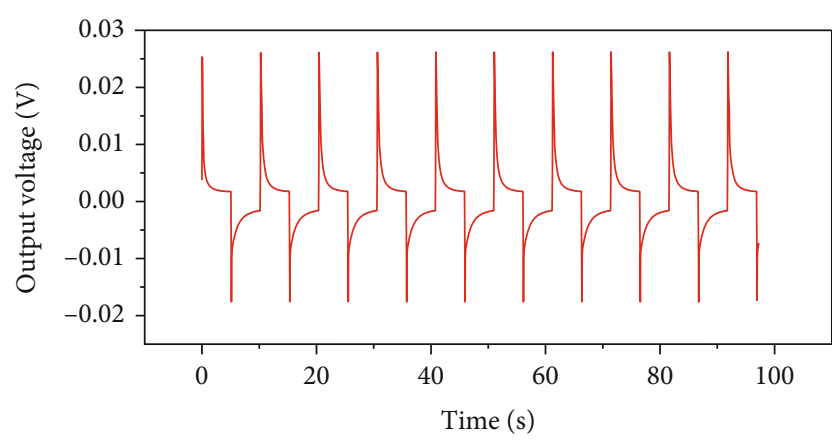

Figure 5: Output voltage plot for eye-movement frequency.

that the proportion of time spent with eyes closed within a blink cycle was too large. Thus, it can be judged that the subject was in a state between semiconsciousness and fatigue. It is important to note that the actual test cannot be judged from a single blink or a few blinks but requires a large amount of continuous data collection and analysis. The voltage curve shown in the results has a clear cut-off point, with the frequency and amplitude of blinks in the positive yield range for the first few segments and a sudden increase in blink frequency for the latter segments.

4.2. Optimal Characterization of Ceramic Processes. When users evaluate a product, their cognitive activities are based on intuition, and when they observe the details of the product, they are often only interested in a certain part, so the method to be used when testing users is different from that used for experts or professional product designers. In order to ensure the accuracy of the evaluation, other subjective testing methods should be provided for the tested users. To make subjective tests more user-friendly, tests can be conducted in the form of easy-to-answer scoring scales. In recent years, the measurement technique of eye-movement behavior has been more intensively studied. Eye-movement measurement techniques, as well as eye-movement data collection and analysis techniques, have been more mature and have been widely used in other fields; some physiological indicators, such as brain waves, are very complicated in their mechanism of action, and although research on these physiological indicators has achieved certain results, they are still in the superficial application stage, and there is still a certain distance from the in-depth and extensive application. To sum up, the measurement of physiological data in this paper adopts the eye-movement measurement technique.

Blinking is a rapid eye closing movement called the transient reflex. There are usually two types of blinks: one is involuntary blink movement; the other is reflexive eye closure movement. The involuntary blinking movement, which is usually done without external stimuli except for inflammatory and painful stimuli, is an involuntary blinking movement that people accomplish unconsciously is actually a protective action that distributes tears evenly across the cornea and conjunctiva to keep them moist, so the upper eyelid moves much more than the lower eyelid during the blinking process. Therefore, in order to maximize the true blink pro- cess, we chose to attach the sensor to the epidermis of the upper eyelid.

In order to obtain the maximum strain at the upper eyelid, we performed a simple tracing experiment. First, we traced the dot at the upper eyelid, then fixed the relative positions of the camera and the pupil, and took pictures of the fully open and fully closed layers, respectively, and superimposed the two layers to roughly measure the maximum strain direction at an angle of approximately $45^{\circ}$ from the horizontal. Therefore, we finally chose to cut the piezoelectric flexible sensor into $2 \mathrm{~cm} * \mathrm{~cm}$ size and paste it between the upper eyelid and eyebrow at an angle of $45^{\circ}$. The bonding material used in the experiments was medical double-sided adhesive, which is thin, breathable, highly biocompatible, and highly adhesive. First, we made the subject blink normally with a blink cycle of about $0.4 \mathrm{~s}$, and the voltage curve of the output voltage after filtering the spurious waves at $15 \mathrm{~Hz}$ is shown in Figure 6. $0.2 \mathrm{~s}$ is consistent with the data in medical literature. This time period is particularly important in fatigue driving monitoring, because it represents the "interference zone" in the driving process.

For example, when driving on a highway at $120 \mathrm{~km} / \mathrm{h}$, $0.5 \mathrm{~s}$ of eye closure means a blind driving distance of 16.5 meters, and a slight steering in such a long distance can lead to a serious traffic accident. If we take a certain period of the blink cycle in the graph and zoom in on the time axis, we can get this result. From the result, we can clearly see that the voltage curve in one cycle can be divided into four parts: the first is the rising voltage section, which means that the piezoelectric film receives a positive strain change and stops after reaching the peak, and it represents the time period from opening to closing of the eye; the second section is the voltage drop from the peak to zero, which represents no strain change in the piezoelectric film; the polarized charge discharges naturally with the circuit, and this process reacts in the blink as the eye remains closed; the next stage is the reverse voltage increase to the pole, which means the piezoelectric film receives a reverse strain change; that is, the eyelid gradually opens from closed; the last stage is the voltage returns to zero from the reverse pole and keeps the voltage at zero until the next blink cycle, which is also the zone where the piezoelectric film is not subject to strain change after free discharge, and it represents the gaze zone that keeps the eyes open.

Technology continues to develop, and ceramic production technology gradually has a trend of assimilation, so ceramic product modeling will be an important factor in attracting users. Design concept is the soul of ceramic product modeling; whether it can effectively convey to the user through the product modeling is one of the key factors in the success of ceramic product modeling. In the ceramic product modeling design, the designer's expression of the product design concept is always running through it. These design concepts will eventually be conveyed to the user through the intermediate carrier of ceramic product modeling. At the same time, the user through the cognition of the product shape will produce an understanding of its design concept, called the perceptual experience. Therefore, around the product design concept, the consistency of the two ends 


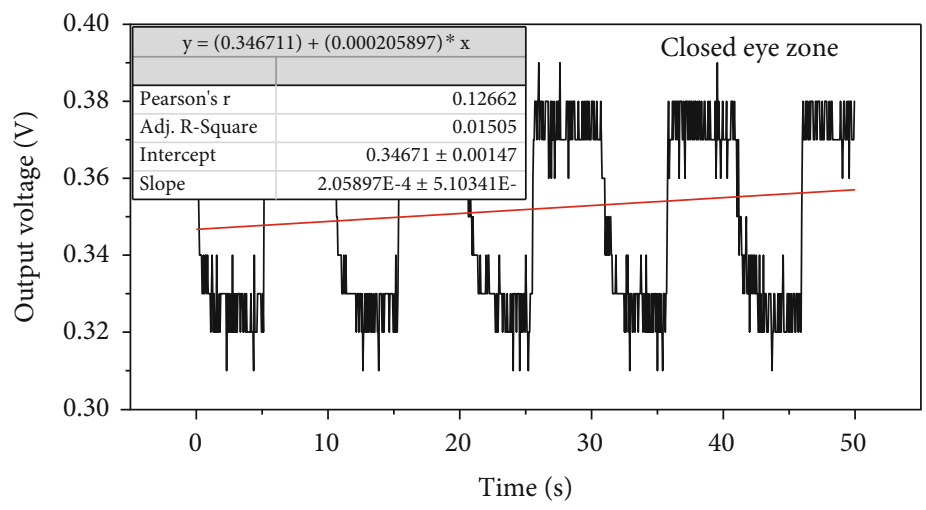

Figure 6: Output voltage of the blink cycle.

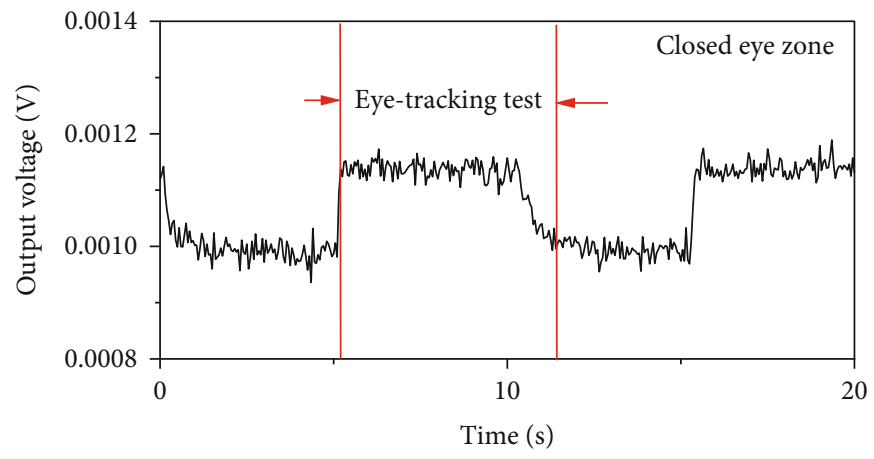

FIGURE 7: Eye tracking data feedback.

of the designer's expression and the user's perceptual experience determines the success of the product design. However, influenced by the differences in factors such as the knowledge background of designers and users, the expression of the designer's design concept may not always be accurately conveyed to the user to produce the same perception. Therefore, for product styling, a method that can relatively accurately evaluate the degree of consistency between the designer's design expression and the user's perceived experience is necessary. Product design concepts are highly emotional and difficult to quantify, so it is difficult to evaluate the consistency between the designer's design expression and the user's perceived experience.

Therefore, the evaluation method based on the theory of semantic perception is relatively reasonable and operable. By refining the designer's product design concept, the target users are asked to evaluate the perceptual experience of the product, so as to compare and evaluate the degree of consistency between the designer's design expression and the user's perceptual experience. In the process of obtaining the user's perception and evaluation of the product design concept, if we only rely on subjective testing, the test results will be more arbitrary, and the user will give inaccurate results due to forgetfulness and other reasons, and the authenticity of the results will be affected. Therefore, it is necessary to combine the methods of objective testing to reduce the error of subjective testing. With the development of eye-tracking technology in recent years, eye-tracking test is gradually used in the field of perceptual analysis as a method to measure objective physiological responses. In this paper, eye tracking test is used as an objective means to evaluate the consistency between designers' design expressions and users' perceptual experiences, and an evaluation method combining semantic questionnaire and eye tracking technology for ceramic product modeling design is proposed, as shown in Figure 7. There are many disadvantages of using physical products for eye-tracking experiments, so this paper uses a computer to display photos of the tested ceramic products, firstly presenting the product pictures to the users and asking them to fill in the relevant semantic perception questionnaire to clarify their semantic perception of the product design concept; then carrying out a local modeling semantic association eye-tracking test, this test separately asks the users to perceive the semantic words of the tested products in the previous step. After the eye-tracking test, the subjects were asked to fill in the semantic association questionnaire; finally, the data of the two questionnaires and the eye-tracking test were collated from all the users, and their consistency was compared and analyzed, and the results of the comparison and analysis were given to the subjects to obtain further updates of the users' semantic perception evaluation of the product.

In this paper, a questionnaire was used to record user perceptions when evaluating the shape of ceramic products. In the analysis of ceramic products, the semantics of the concept related to the design of the product were selected and tested, and interfering semantics of the design concept and other semantics used to evaluate ceramic products were 


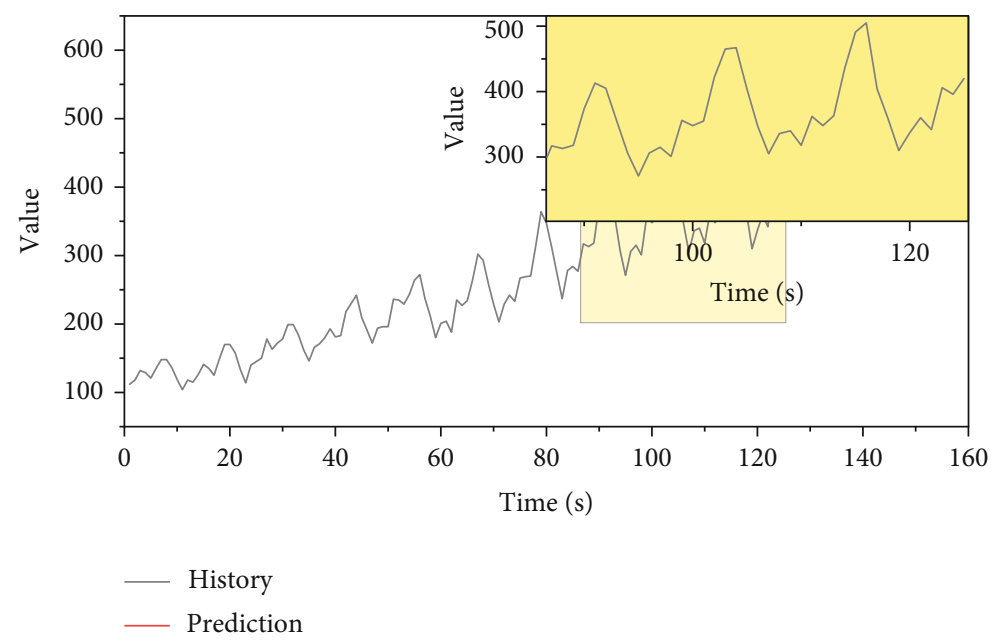

Figure 8: Correlation between eye-movement data and ceramic mechanics.

added to make the user perception evaluation more comprehensive. The interference words used in the test are words with the opposite meaning of the design concept semantic words of the product under test, which can be used to analyze the level of recognition of the design concept semantic words when facing the product under test; by comparing the perception of interference words and semantic words, the level of semantic perception of the user when facing the product under test can be clarified. The eye-tracking test and questionnaire test were implemented to record the users' perception of the product styling design concept, and thus, the test results were obtained. The experimental materials for both tests are the same, and both are frontal photos of the tested products, which allow users to observe partial styling and record the perception. After presenting the product shape picture to the user, the user fills in the product shape perception semantic questionnaire. The user is asked to select no more than a certain number of semantic words among the semantic words provided in the questionnaire to evaluate the semantic perception of the tested product. If the subject perceives other semantic words in addition to the given semantic words, he/she can fill in the corresponding positions in the questionnaire.

At the same time, the user is required to score the product styling on a scale of $0-100$ based on all the semantic words selected or filled in, respectively, to evaluate and analyze the effectiveness of the design concept communication. The test task of eye tracking is the semantic vocabulary selected or filled in by the user in the first questionnaire. For each semantic term, the user observes the product image and looks at the partial shape where the semantic term can be perceived. The eyetracker will record the user's eye movements during the perception process, as shown in Figure 8. After the eye-tracking test, the hotspot maps of the user's gaze on each semantic term are extracted. The eye-tracking device can record the user's eye-movement behavior, so the test results may not be exactly the semantic perception results but may be the local location that the user pays more attention to. At the same time, users' evaluation of the semantic perception of product styling is usually more random, and there is a tendency for inconsistency between two evaluations. The eye-tracking device can objectively record the product localization that the subject instinctively looked at when hearing a semantic word, and its results can supplement the shortcomings of the questionnaire test. However, interviews with users are still needed to compare the eye-movement test data with the questionnaire data and then to clarify the correlation results. Compared with other categories, although ceramic modeling has relatively abstract formal characteristics, but it is the extension of the modeling, still has obvious morphological characteristics, and the impact on people's aesthetic mood.

After completing the above experiments, all subjects' eye-movement information was analyzed and collected and organized using the accompanying professional software, Tobii Pro Lab. The polygon tool in the AOI module of the software was used to divide the areas of interest into areas of interest for the four products in the eye-movement experiment, and then, the eye-movement data from each area were collected, exported, and saved as Excel files. The Tobii Pro Lab software enables automatic superimposition of oculomotor data information, allowing the display of 4 oculomotor data from 4 products and saving hotspot maps. Based on the literature, four eye-movement indicators, namely, total gaze time, first gaze duration, number of visits, and number of gaze points, were selected for analysis. The results of the Pearson correlation analysis showed that there was a positive correlation between all four eye-movement data and scoring values. According to the literature, the duration of first gaze is similar to the priority effect in the field of psychology, in that when a subject is interested in a certain area, his or her eyes will be attracted to that area first and stay there; the number of gaze points in a certain area often represents the intensity of the subject's interest in that area. The number of visits means the number of eye jumps from other areas to the area of interest. Usually, the subjects' eye-movement evaluation activity of the experimental tested product is the observation and comparison activity of several 
tested products; if the subjects like one of the tested products more, then the product tends to become a benchmark for comparison, and the more visits will be made.

\section{Conclusion}

In this paper, by implementing the overall ceramic product styling evaluation experiment, we obtained the eye-tracking data and user scoring data during the evaluation activity; used the support vector machine learning method to establish a mathematical model, which mainly involves the mapping relationship between the eye-tracking data and the user scoring value; and verified the validity of the model using the test set data. The results of the validation show that the support vector machine classification method can effectively establish the overall evaluation model of ceramic product modeling based on eye tracking and provide an objective basis for ceramic product modeling evaluation. The application of semantic perception evaluation of ceramic product modeling based on eye-tracking was implemented. A ceramic cultural and creative product of a brand was used as the test material, and the target user was used as the test object, and the semantic questionnaire test of ceramic product modeling perception, the eye-movement test of modeling semantic perception, and the questionnaire test of local modeling semantic association were conducted in turn. In addition, the user's eye movement test results were compared with the questionnaire test results. The user interview method is used to obtain the user's perception of the product design concept and analyze the semantic expression of each part of the product and evaluate the effectiveness of the product modeling design concept. In the environment of rapid development of the consumer market, users' needs for products are constantly changing, and users are gradually taking the leading position in the consumer market. In the case of product homogenization, whether the product shape is attractive to users becomes crucial, and the user-oriented product shape evaluation method will be more widely used.

\section{Data Availability}

The data used to support the findings of this study are available from the corresponding author upon request.

\section{Conflicts of Interest}

The authors declare that they have no known competing financial interests or personal relationships that could have appeared to influence the work reported in this paper.

\section{Acknowledgments}

This work was supported by the Hubei University of Technology.

\section{References}

[1] P. Kasprowski and K. Harezlak, "Fusion of eye movement and mouse dynamics for reliable behavioral biometrics," Pattern Analysis and Applications, vol. 21, no. 1, pp. 91-103, 2018.
[2] P. D. Gunawardane, R. R. MacNeil, L. Zhao, J. T. Enns, C. W. de Silva, and M. Chiao, "A fusion algorithm for saccade eye movement enhancement with EOG and lumped-element models," IEEE Transactions on Biomedical Engineering, vol. 68, no. 10, pp. 3048-3058, 2021.

[3] W. Dong, L. Yang, R. Gravina, and G. Fortino, "ANFIS fusion algorithm for eye movement recognition via soft multifunctional electronic skin," Information Fusion, vol. 71, pp. 99-108, 2021.

[4] T. Li, J. Yang, D. Bai, and Y. Wang, "A new directional intention identification approach for intelligent wheelchair based on fusion of EOG signal and eye movement signal," in IEEE International Conference on Intelligence and Safety for Robotics, pp. 470-474, Shenyang, China, 2018.

[5] T. Otomo, S. Mochiduki, E. Ishii, Y. Hoshino, and M. Yamada, "Analysis of eye movement and critical fusion frequency responses to different movie types," IEICE Transactions on Fundamentals of Electronics, Communications and Computer Sciences, vol. 102, no. 9, pp. 1254-1258, 2019.

[6] C. T. Kuo, P.-W. Chi, V. Chang, and C.-L. Lei, "SFaaS: keeping an eye on IoT fusion environment with security fusion as a service," Future Generation Computer Systems, vol. 86, pp. 14241436, 2018.

[7] G. Orlandi, R. Calvini, G. Foca, L. Pigani, G. Vasile Simone, and A. Ulrici, "Data fusion of electronic eye and electronic tongue signals to monitor grape ripening," Talanta, vol. 195, pp. 181-189, 2019.

[8] S. C. Mehta, R. F. Kelley, and D. B. Tesar, "Protein conjugates and fusion proteins as ocular therapeutics," Drug Discovery Today, vol. 24, no. 8, pp. 1440-1445, 2019.

[9] J. Choi, S. K. Lee, and S. Park, "Electromagnetic tracking-based ultrasound/computed tomography fusion imaging in dogs: preliminary application to ocular and periocular regions," Journal of Veterinary Science, vol. 20, no. 5, pp. 1-8, 2019.

[10] Y. Lv, W. Zhou, J. Lei, L. Ye, and T. Luo, "Attention-based fusion network for human eye-fixation prediction in 3D images," Optics Express, vol. 27, no. 23, pp. 34056-34066, 2019.

[11] Y. Sun, P. Yan, Z. Li, J. Zou, and D. Hong, "Driver fatigue detection system based on colored and infrared eye features fusion," Computers, Materials \& Continua, vol. 63, no. 3, pp. 1563-1574, 2020.

[12] A. Arunkumar, D. Surendran, and S. Sreya, "Data fusion and machine learning in medical diagnosis: a bird eye view," Journal of Computational and Theoretical Nanoscience, vol. 16, no. 12, pp. 5127-5133, 2019.

[13] S. Xia, M. Liu, C. Wang et al., "Inhibition of SARS-CoV-2 (previously $2019-\mathrm{nCoV}$ ) infection by a highly potent pancoronavirus fusion inhibitor targeting its spike protein that harbors a high capacity to mediate membrane fusion," Cell Research, vol. 30, no. 4, pp. 343-355, 2020.

[14] M. Mauthe, I. Orhon, C. Rocchi et al., "Chloroquine inhibits autophagic flux by decreasing autophagosome-lysosome fusion," Autophagy, vol. 14, no. 8, pp. 1435-1455, 2018.

[15] A. Drilon, T. W. Laetsch, S. Kummar et al., "Efficacy of larotrectinib in TRK fusion-positive cancers in adults and children," The New England Journal of Medicine, vol. 378, no. 8, pp. 731-739, 2018.

[16] F.-C. Chen and M. R. Jahanshahi, "NB-CNN: deep learningbased crack detection using convolutional neural network and Naïve Bayes data fusion," IEEE Transactions on Industrial Electronics, vol. 65, no. 5, pp. 4392-4400, 2018. 
[17] J. Ma, W. Yu, P. Liang, C. Li, and J. Jiang, "FusionGAN: a generative adversarial network for infrared and visible image fusion," Information Fusion, vol. 48, pp. 11-26, 2019.

[18] C. O. Ancuti, C. Ancuti, C. de Vleeschouwer, and P. Bekaert, "Color balance and fusion for underwater image enhancement," IEEE Transactions on Image Processing, vol. 27, no. 1, pp. 379-393, 2018.

[19] E. Cocco, M. Scaltriti, and A. Drilon, "NTRK fusion-positive cancers and TRK inhibitor therapy," Clinical Oncology, vol. 15, no. 12, pp. 731-747, 2018.

[20] R. C. Doebele, A. Drilon, L. Paz-Ares et al., "Entrectinib in patients with advanced or metastatic NTRK fusion-positive solid tumours: integrated analysis of three phase 1-2 trials," The Lancet Oncology, vol. 21, no. 2, pp. 271-282, 2020.

[21] S. Xia, Y. Zhu, M. Liu et al., "Fusion mechanism of 2019-nCoV and fusion inhibitors targeting HR1 domain in spike protein," Cellular \& Molecular Immunology, vol. 17, no. 7, pp. 765-767, 2020. 